\title{
Hybrid Overlay Networks Management for Real-Time Multimedia Streaming over P2P Networks
}

\author{
Mubashar Mushtaq and Toufik Ahmed \\ CNRS LaBRI Lab. - University of Bordeaux 1 \\ 351 Cours de la Libération, Talence Cedex 33405 - France \\ \{mushtaq, tad\}@labri.fr
}

\begin{abstract}
Recent growth of the multimedia content delivery over the Internet and the popularity of the peer-to-peer (P2P) architecture have opened new horizons for emerging novel services over the Internet. Currently, most of multimedia services are being offered to the end users by using set-top boxes installation on the client's premises, with integrated media storage capabilities and their adaptation. The organization of the end-clients in P2P fashion has great potential to change business models to offer new value-added multimedia services and therefore to generate substantial revenue for service providers. In this paper, we present a mechanism to organize the sender peers in hierarchical hybrid overlay networks. The objective of such organization is to facilitate the receiver peer (content consumer) to select best sender peers for the provision of better QoS (Quality of Service). To construct the hybrid overlay networks, peers offering the same video quality are placed together at the same level of overlay networks. The organization of sender peers within these overlays is subject to (1) the semantic of the video provided by the peer (base layer, or enhancement layers) and (2) the QoS offered by each peer along the end-to-end path. The proposed streaming mechanism is receiver-centric where receiver peer selects a number of sender peers from the overlay networks to receive media contents. The performance evaluation performed using ns-2 simulator shows that hybrid overlays organization mechanism is helpful to enhance the overall QoS by significant improvement in received video packets throughput, the packets drop ratio and transmission delay.
\end{abstract}

\section{Introduction}

P2P networking frameworks have recently received a lot of attention by the research community, as they provide an efficient infrastructure to use available networking resources in a more transparent, scalable and cost-effective way. P2P networks were initially designed and were considered suitable for huge contents distribution across networks. These networks possess distributed, scalable, cost-effective, cooperative resource sharing, self-organizing, and many more characteristics that have encouraged service providers (SPs) to deploy many real-time applications over large scale heterogeneous networks. This is considered as a giant shift of technology that has changed the way people interact with the technologies. The most important real-time 
applications based on P2P framework include: video streaming, video on demand, P2P based IP-TV, P2P gaming, signaling for IP telephony, etc. Overlay networks are considered as the most promising infrastructure for the deployment of distributed applications. Many existing multimedia applications over P2P networks are based on the organization of the participating peers in overlay networks. Many research developments have been observed overlay networks organization that address the scalability, efficient management, and self organization of overlay networks. The well known overlay networks like Content Addressable Network (CAN) [1], Chord [2], and Pastry [3] have been proposed for the Internet routing to guarantee better quality of service.

On the other hand, recent advances in audiovisual content coding have favored a widespread growing of multimedia streaming and Video on Demand (VoD) services. A number of home holders are subscribing to these services by the service provider through a set-top box. These boxes are generally equipped with specific functions such as storage space, on which the client can store programs, movies, and other desired contents. The service provider has full access for indexing, tracking and to use the contents available in these set-top boxes. These characteristics enable service providers to use such boxes as independent content servers. In the rest of paper, we refer these boxes as peers when used in P2P networks. The terms set-top-box and peer are used interchangeably. In fact, most of these boxes are capable of performing media content adaptation in accordance with the end client's requirements. These features extend the usability of these boxes to that of media servers for the end clients possessing different characteristics, i.e. heterogeneous clients. Another point of heterogeneity is related to the end-user connection. Most of the end-clients (service subscribers) connect to the network using ADSL (Asymmetric Digital Subscriber Line) or CMTS (Cable Modem Termination System), where uplink capacity is 3-8 time lesser than that of the downlink. If media content is requested from a single box, it cannot be achieved. In fact, a single peer might not be able to meet the requirements of any one request. In this regard, we propose to select multiple sender peers to cooperate in the streaming mechanism using multi-source streaming. The use of multiple peers for the service delivery reduces the load on the central server, reduces the start-up delay, latency, and improves the overall Quality of Service (QoS) of the system.

In this paper, we present a quality adaptive mechanism for the multimedia streaming and video on demand services over P2P networks. This mechanism is based on the organization of sender peers in hybrid overlay networks. These overlay networks are formed on the basis of offered video quality and end-to-end probing among the sender and receiver peers.

The network topology for multimedia streaming and video on demand services is illustrated in the Fig. 1 where different service clients with variant characteristics are connected to the network. In this topology, we consider only those peers which have the requested media contents and are willing to participate in the streaming mechanism. The receiver peer selects multiple sender peers to receive the media contents from the overlay networks. The received media contents are combined to decode the received video with a higher quality. The sender peers are selected from the different overlays on the basis of receiver characteristics and its preferences. 


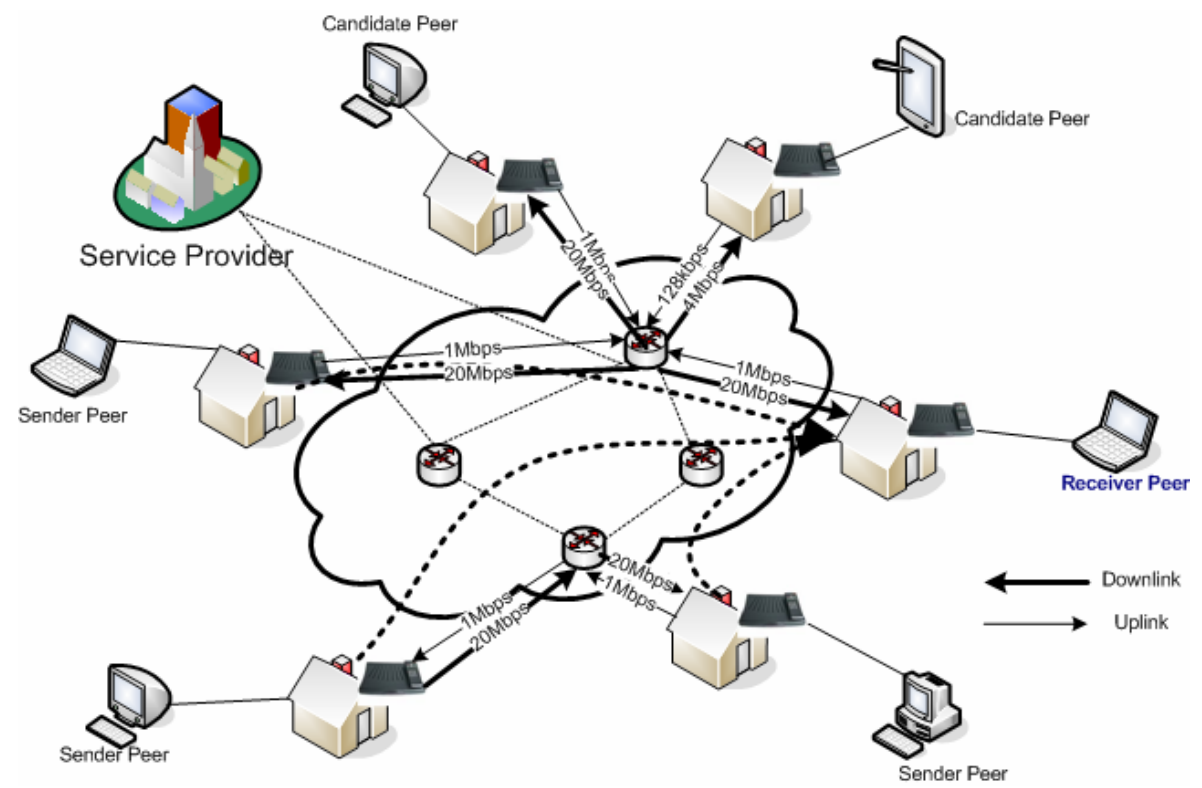

Fig. 1. Network Topology for Multimedia Streaming / Video on Demand

The rest of the paper is organized as follows. A brief motivation and some of the related work is presented in the section 2. Section 3 describes an overview of the scalable video coding (SVC) scheme. The proposed streaming mechanism based on the organization of sender peers in hierarchical overlay networks is given in section 4. The evaluation results of proposed mechanism using simulations are presented in section 5. Finally, concluding remarks are presented in section 6 .

\section{Motivation and Related Works}

There have been significant studies carried out in the context of multimedia streaming applications and for the construction of overlay networks to enhance the QoS. Most of these studies have investigated and proposed solutions for the efficient routing algorithms, organization techniques, and self organization.

Zhang et al. [4] developed a framework for live media streaming that is based on data-driven overlay networks where each node periodically exchanges data availability information with other nodes. The management of nodes which join the networks is controlled by an origin node that is persistent during the life time of the streaming session. In the proposed mechanism, a scheduling algorithm is devised for heterogeneous clients. The nodes in the data-driven overlay network are organized in a Breath-First-Search (BFS) tree, where origin node is located at level ' 0 ' while any node at level ' $\mathrm{k}$ ' can be reached in ' $\mathrm{k}$ ' hops from the origin. 
Tian et al. [5] presented a framework for the Hybrid Overlay Structure that is based on Random Walks. The proposed organization is locality-aware where nodes are organized in different clusters. Crespo et al. [6] proposed the "SON" mechanism for the organization of different nodes. The proposed mechanism is based on the semantic based organization of nodes. Nodes having the same type of media files are organized close to each other and therefore clustered together. The semantic based organization result into improvement of the system performance in terms of the efficiency in query search.

The organization of peers (nodes) in overlay networks systems also has been presented in research work such as CAN [1], CHORD [1], Pastry [2], and Tapestry [7]. These systems provide efficient routing mechanism for the structured $\mathrm{P} 2 \mathrm{P}$ overlay networks. The major proposed solutions organized peers in overlay networks on the basis of distances, i.e. Locality-aware. The motivation behind our proposed mechanism is to use the overlay networks for the best sender peers selection in order to support heterogeneous clients and to improve the QoS while efficiently utilizing the available network resources. In our proposed hybrid overlay networks mechanism, we organize different sender peers in different hierarchical overlay networks considering both (1) the semantic of the video and (2) the QoS offered by each peer. The detailed hybrid overlay organization mechanism is presented in section 4 .

\section{Scalable Video Coding (SVC)}

Multimedia content adaptation is considered as an important technique to provide QoS management for multimedia delivery over heterogeneous networks to meet the requirements of clients with distinct characteristics. Many video encoding schemes have been proposed for real-time applications operating for heterogeneous networks and terminals. Layered encoding and multiple description coding [8] are considered suitable for many applications including P2P services. At present, Scalable Video Coding (SVC) is considered most promising video encoding format for streaming application in heterogeneous networks and terminals [9]. A scalable video coding is used to produce highly compressed bit-streams, to generate a wide variety of bit-rates. An original SVC stream can be truncated to produce videos of different qualities, resolutions, and frame rates using respectively SNR (signal-to-noise ratio), spatial, and temporal scalabilities.

In this paper, we focus on the use of SVC video formats as an important component of the proposed $\mathrm{P} 2 \mathrm{P}$ system while organizing the sender peers in hybrid overlay networks. The SVC characteristics make it more suitable for heterogeneous environments especially P2P networks. A layered stream representation of SVC in terms of spatial, temporal, and SNR resolution is shown in Fig. 2. In SVC encoding scheme, each quality tier is decodable with different characteristics. The first tier providing the basic quality of the video is called "Base Tier" while other tiers which are used to enhance the overall video quality of the base tier are called "Enhancement Tiers" [10]. 


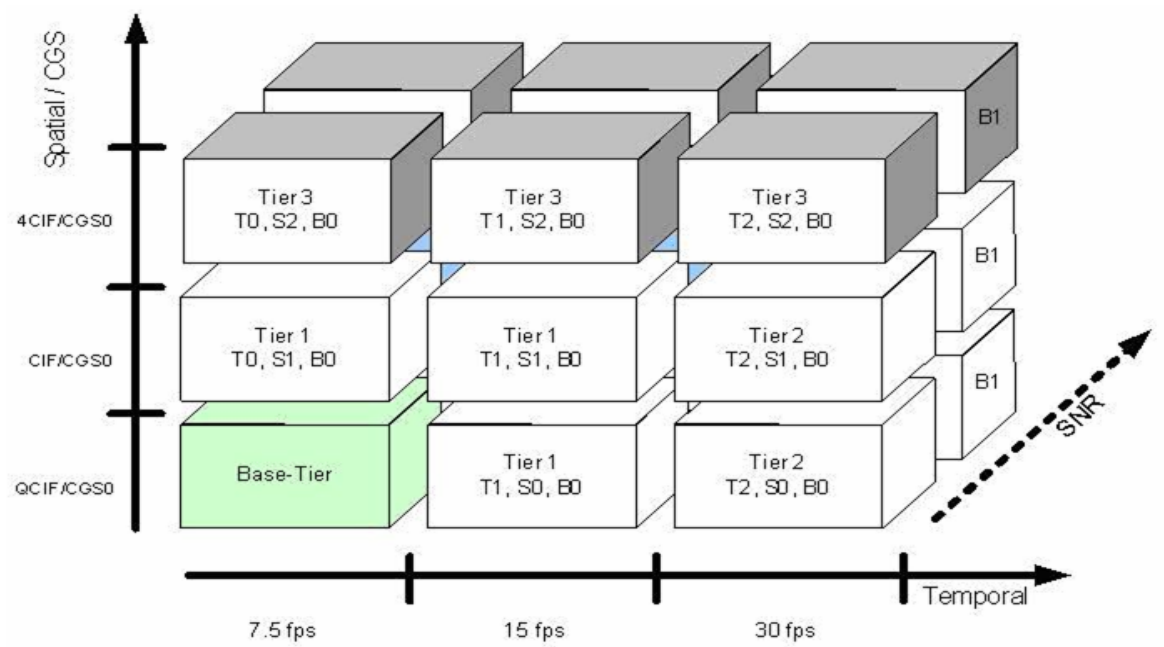

Fig. 2. Scalable Video Encoding - A Layered Stream Representation

\section{Hybrid Overlay Networks Organization: Semantic and QoS-Awareness}

In this section, we describe our proposed multimedia streaming mechanism over P2P networks. Our targeted P2P streaming solution is based on the collective communication among multiple senders towards a single receiver where the receiver peer orchestrates the overall streaming mechanism. Receiver peer maintains the list of the entire candidate peers which have the requested media contents and are ready to participate in the streaming process. A peer is considered as a candidate peer if it has the requested contents and it is willing to participate in the streaming mechanism. The sender peers are organized in different hybrid overlay networks based on the offered content (i.e. which SVC tier is provided per peer) and based on the QoS offered by the peer. The organization of peers in hybrid overlay networks and their management is described in the following sub-sections.

\subsection{Overlay Networks Organization}

Many overlay organization schemes have been proposed to address different issues including efficiency, scalability, self-organization, efficient routing. For this study, we have slightly different objective for the organization of the sender peers in overlay networks. We aim to facilitate the receiver peer to select the best sender peer to enhance the overall QoS for the offered services. In this paper, we organize the sender peers in hierarchical hybrid overlay networks. This hybrid peer organization is based on two important characteristics, (1) semantic aware peer organization, (2) quality aware or locality-aware peer organization. The main goal for this hybrid based organization is to bring sender peers closer to the receiver peer and to provide an efficient mechanism for the selection of best peers with the required video quality. 
The overlay networks can be classified on the basis of peers' organization criterion. Here, we described some of these classifications along with our proposed Hybrid peers organization.

- Locality based peer organization: Locality based peer organization is performed on the basis of geographical/physical distances between sender and receiver peers. The locality of peers is determined by different parameters, for example, Euclidean distances, logical distances estimated using number of intermediate peers (e.g. number of Hops, TTL measurement, etc.), and using end-to-end round trip time (RTT) measurements. Locality-aware or QoS-aware techniques primarily intend to reduce the delays by minimizing the logical distance between the source peers and the receiving peers for each service request. Such locality based peers organization results in the minimizing of start-up delay and latency during the streaming mechanism. Our mechanism is based on RTT measurement as an indicator for the quality of the link between the sender and the destination.

- Semantic based peer organization: The structure of media contents is useful for the selection and organization of the appropriate peers in overlay networks. In semantic based organization, peers with the same media contents or offering the same video quality are placed together in overlay networks. Semantic based peers organization influences the delivery of important parts of the audiovisual content to the important peer. Such organization makes it convenient to search media contents rapidly. Semantic based peer organization can be a good choice for scalable video coding and in other coding schemes where media contents are distinguished according to priority. Our mechanism is best adapted to SVC coding or any schemes that can provide priority between sub-streams.

- Hybrid peer organization: We propose hybrid peers organization that is influenced by combining locality based (or quality-based) organization and semantic based organization schemes. In this hierarchical hybrid overlay organization, sender peers are arranged in hierarchical overlay networks. The streaming mechanism is receiver-centric. The sender peers offering the same video quality tiers of the same media types are placed at the same level in overlay networks. Sender peers within each level of overlay networks are organized according to locality-aware peer organization. We use round-trip-time "RTT" as an indicator of the offered QoS by each peer. In fact, the best peer is the one offering the best QoS (i.e. the lower RTT). This peer should be selected by the receiver to contribute the base tier of the video. In [11], we have noticed that "RTT" can be used to improve the overall QoS for the streaming applications in heterogeneous networks like P2P. The motivation behind using RTT is its relationship with the offered bandwidth on the end-to-end path. A simplified version of the equation given the bandwidth (data rate) of a particular TCP-like session is given in Eq. 1. The MTU represents the maximum transfer unit and the "Loss" represents the packet loss rate [12].

$$
\text { Bandwidth }=1.3 * M T U /(R T T * \sqrt{\text { Loss }})
$$


It is commonly agreed that the lower the "RTT" the more the offered data rate (bandwidth) is higher (c.f. Eq. 1). Thus, peers offering lower RTTs are considered as having a higher available bandwidth and that may be shared fairly among the services. This leads us to propose an efficient peer organization mechanism based on active measurement of peers. Any peer is considered as an important peer if it provides the lowest "RTT" and is assigned to contribute the SVC base video tier.

A hybrid organization of the sender peers in different hierarchies is illustrated in Fig. 3. Here, sender peers are divided into three different levels of overlay networks. The organization of these peers is carried out on the basis of the "RTT" probing and on the basis of offered video quality tiers. Overlay 1 consists of the peers offering the base SVC tier quality of video, overlay 2 and overlay 3 depicts the peers offering enhancement SVC tier 1 and enhancement SVC tier 2 respectively. Within each overlay network, peers are organized according to the end-to-end RTT probing carried out between the receiver and each sender peer. We exploit the "MinHeap" Tree structure for the organization of peers. A "MinHeap" is a minimum complete binary tree where the value in each node is less or equal to those in its children. In such tree structures ' $n$ ' peers can be organized in $\Theta(n \log n)$ time. The organization of peers inside the "MinHeap" tree structure is shown in Fig. 3. The peer offering minimum "RTT" is always present at the root of the overlay tree and provides an efficient mechanism to select the best peer offering the best.

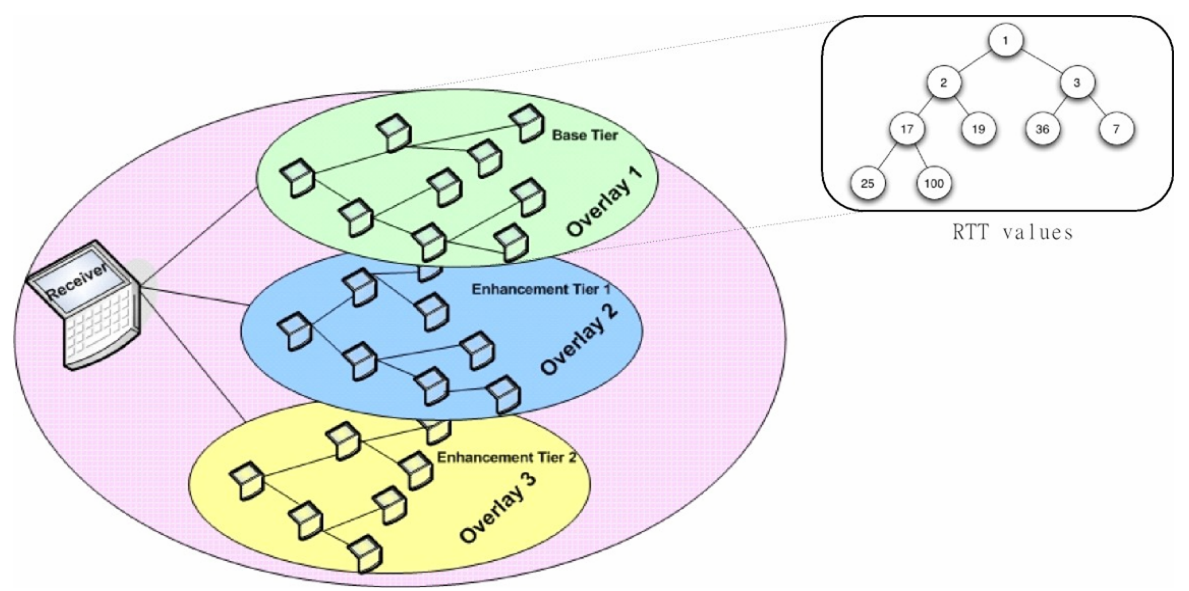

Fig. 3. Layered Organization

\subsection{Management of the Sender Peers}

The main components of the P2P based media streaming and video on demand services include: indexing of the media contents available to each sender peer, 
signaling among the peers for their activation, organization of sender peers in virtual overlay networks, media contents retrieval, selection of best sender peer to serve for subsequent request, sender peers switching, selection of quality adaptation path, etc..

In this paper, we did not discussed the issues of indexing, signaling, and data retrieval mechanisms and we are more concerned with the organization of peers in overlay networks for the efficient sender peers selection to enhance the overall QoS.

The management of senders peers in the P2P streaming mechanism is essential as (1) a single peer is not able to serve alone a service request due to its uplink capacity limitations, (2) some selected peers might leave the P2P network at any moment - for example, when the end-user may switch off its system, and (3) additional contributions from new source peers improves the overall Quality of Service (QoS).

- Selection and Switching of Sender Peers: We propose to select multiple sender peers from different overlay networks to address the aforementioned issues. We described earlier that scalable video coding is used for video encoding schemes, thus the selection of the best peers is performed by assigning the best quality tier (Base tier) to the best sender peer. A peer is consider as the best peer if it offers the lowest end-to-end "RTT" to the destination. The peers are organized using the "MinHeap" tree structure in different overlay networks thus the best peer in each overlay is located at the root of the "MinHeap" tree that is accessible to the receiver peer in $\Theta 1$ time.

P2P networks possess highly dynamic nature and show unpredictable behavior; any peer node can enter or leave the network without any prior notification. In this situation, P2P architecture is not considered reliable for multimedia streaming applications which require permanent connection establishment throughout the streaming process. During long streaming sessions, network conditions can change drastically. This may involve many factors among which (1) a sender peer crashes and /or stops contributing to the media content, (2) a shared bandwidth is changed since the network conditions change, (3) some new peers enter the network and provide better bandwidth sharing and low "RTT" value, (4) heavy traffic on a particular peer can cause more packet loss, and (5) high inter packets delay ultimately degrades the overall QoS.

Network fluctuations degrade received video quality dramatically. Such network fluctuations can be detected using the "RTT" measurements. If "RTT" values become high for a certain sender peer, the sender peer assumes that network links are no longer suitable for the streaming session. This issue is handled by the switching mechanism in two ways. First, if there are some peers available with low "RTT" values in the subset of tracked peers, the effected peer could be replaced with this peer to maintain a smooth video packet delivery. In other case, if no other best peer is available, stream switching is enforced instead of peers switching. In stream switching, receiver peer re-adjusts the received video streams dynamically. This can be done easily using SVC stream organization as it offers threedimensional scalability (i.e. special, temporal and SNR). Thus, the received video quality is adapted to the actual available resources in the network by dropping the streaming of some SVC tiers. We implemented the exponential weighted moving average "EWMA" to prevent from oscillation effects while updating peers after 
each "RTT" calculation. EWMA is an Exponential Smoothing technique [13] that employs one exponential smoothing parameter to give more weight to recent observations and less weight to older observations and vice-versa.

Can the best peer always remain the best? Our targeted architecture for the proposed solution is based on many to one streaming, i.e. a single receiver selects multiple sender peers to receive different parts of the requested media contents. In this scenario, what happens when a number of receivers request for the same media contents? Every receiver peer should prefer to select the best sender peer to receive the contents with the highest quality, in this case due to a certain overload on the best peer; can it still remain the best peer? Nash presented in his classical "Nash Equilibrium" theory [14] that no player can end with higher scores if every player of the certain game choose the best policy. We can state that if every receiver peer intends to select the same best peer, no receiver can get the entire video with good quality and there might be a system crash due to the heavy load (in the case of large number of receiver peers). The best peer for a particular session will not then remain the best for the following session.

The proposed peer organization and their selection provide an efficient way of handling this issue. Firstly, our proposed solution is receiver-centric, and secondly it is based on the probing of sender peers using "RTT" as an indicator of the QoS. We noticed that the probed end-to-end "RTT" is not the same for all the receiver peers, and being the receiver-centric solution every receiver maintains the overlays of sender peers and candidate peers in its own prospective. In our mechanism, the best peer for a receiver might be the worst sender peer for other receiver peer.

- Quality adaptation: Quality adaptation determines the way to assign the video quality tiers to respective sender peers by selecting from different overlay networks. The hybrid overlay organization facilitates to determine the quality adaptation in an efficient manner as all the peers are classified on the basis of offered video quality and QoS. Fig. 4 describes scenarios how a receiver peer can determine quality adaptation based on its preferences. We have presented different quality adaptations patterns (i.e. horizontal adaptation, vertical adaptation, diagonal adaptation, ZigZag adaptation) which represent the patterns to select the sender peers to receive the video quality tiers.
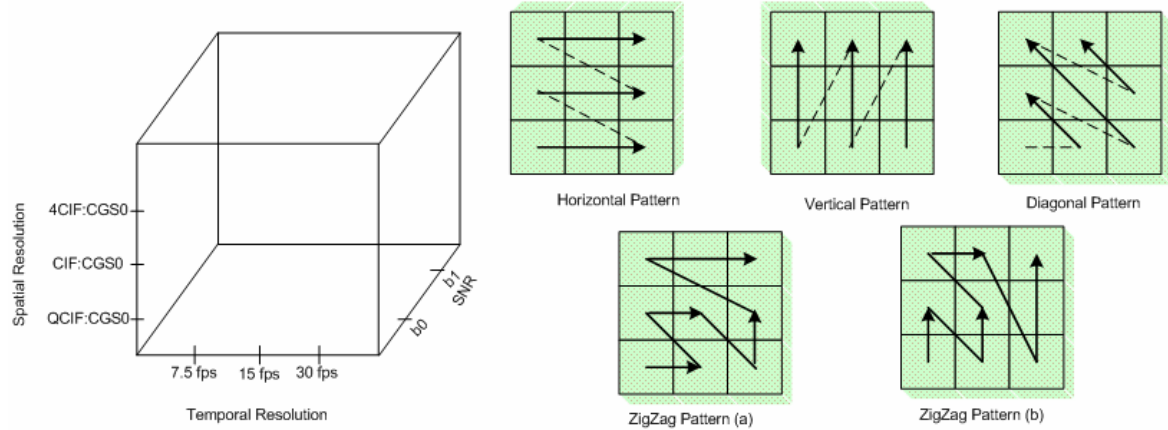

Vertical Pattern

Diagonal Pattern
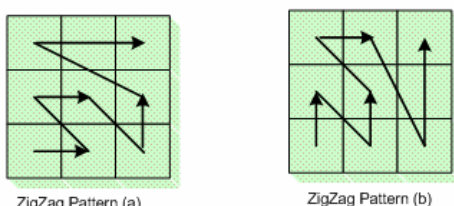

Fig. 4. Quality Adaptation Patterns 
Horizontal and Vertical ordering is more suitable for cases where the receiver intends to receive video quality based on a single parameter, i.e. temporal, spatial, or SNR. ZigZag and Diagonal patterns provide the mechanism to select different sender peers to receive video quality based on multiple parameters. We suggest selecting multiple sender peers from the overlay networks based on ZigZag adaptation for our proposed streaming mechanism. In this way, a better QoS for the received video can be ensured on the basis of available resources.

\section{Performance Evaluation}

This section describes the simulation results for the proposed streaming mechanism performed using the ns-2 simulator.

\subsection{Network Model}

We consider Gnutella like P2P topology for the simulations and we organize different groups of peers on the basis of proposed hybrid overlay scheme. We distribute the SVC tiers equally among different overlays to examine the performance of the proposed mechanism. A receiver peer intends to receive the real-time video packets from multiple sender peers using P2P network. The receiver peers use "RTT" for the probing of sender peers and best peer selection is performed on the basis of lower "RTT". "RTT" values are only indicative that reflect the current network conditions and give sufficient information to choose the best peer.

We perform simulations to receive the video quality of CIF/CGS0 with $15 \mathrm{fps}$. The receiver peer activates a particular sender peer from each overlay group depending on the "RTT" value and the offered video quality based on ZigZag pattern (a) shown in Fig. 4. Each sending peer contributes different quality tiers of the original video file, so that it can be used to reconstruct a video file with the best quality at the receiver node. For the test cases, we generated 4 different quality tiers using MPEG-4 trace files where Base Tier offers $40 \%$ throughput of original video, enhanced tier 1 offers $30 \%$ throughput of original file, enhanced tier 2 offers $20 \%$ throughput of original file and enhancement tier 3 offers $10 \%$ throughput of the original video file.

We noticed that no source peer is providing $100 \%$ throughput of the original video but if a receiver peer receives all the 4 tiers from different sender peers, it is possible to reconstruct the original media file with $100 \%$ quality with the selected characteristics of scalable video coding scheme. The simulation time was 60 seconds and the presented results are the average results of the multiple runs of these simulations. We attached two "CBR sources" to overcharge the network. "CBR source 1" is started at time 5 second and stopped at time 55 second. "CBR source 2" is started at time 10 second, and stopped at time 50. Both sources injected constant throughput of $512 \mathrm{Kbps}$ with 512 Bytes UDP packet lengths. We simulate the adaptation mechanism for two scenarios. 
- Scenario without Quality Adaptation: Simulation without applying any quality adaptation mechanism. In this case, P2P system works as in downloading modes. The sender peers are selected on a random basis.

- Scenario with the proposed Quality Adaptation: Simulation with quality adaptation mechanism is performed by best peer selection and their switching based on the proposed hybrid overlay networks as described in section 4 .

\subsection{Simulation Results}

Fig. 5 shows the received video throughput at receiver peer for both scenarios. We observed that the quality adaptation mechanism improved the received throughput compared to the scenario without quality adaptation. We have noticed few packets drop even with quality adaptation mechanism. These packets drops are caused due to the heavy stress on the network created by CBR/UDP traffic which is presented in Fig. 6. Packet drop ratio is much lesser in the scenario with quality adaptation compared to scenario without adaptation so, our quality adaptation mechanism works fine for this case too. This enhanced throughput and lower packets drops results into the overall improvement in QoS.

We monitored all the active network links constantly and select only one sender peer from each overlay group, i.e. peer having lowest "RTT" and present at the root of "MinHeap" tree. The stream switching is done by selecting the best sender peer offering the better QoS (high bandwidth share). We performed peer switching and implemented EWMA mechanisms to avoid fluctuations caused by peers arrival or removal. Fig. 7(a), Fig. 7(b), Fig. 7(c), and Fig. 7(d) describe the comparison between received and original base tiers, enhancement tier 1, enhanced tier 2, and enhanced tier 3 respectively. We can see clearly that our proposed mechanism performs a smooth video delivery with higher quality, lower loss and delay. A summary of these results is given in Table 1 .

The overall received 1-way packets delay (from sender peer to receiver peer) for both cases is presented in Fig. 8. The packets transmission delay has been significantly improved when quality adaptation mechanism is applied. We reconstructed the expected video file using the "AKIYO" video sequence for the received video quality at the receiver end to evaluate the PSNR (Peak signal-to-noise ratio) objective measurements. The comparison results for PSNR for the videos generated in both scenarios to the original video are shown in Fig. 9.

Table 1. A Summary of Simulation Analysis

\begin{tabular}{|l|c|l|l|l|l|l|}
\hline & $\begin{array}{l}\text { Received } \\
\text { Video } \\
\text { Quality (\%) }\end{array}$ & $\begin{array}{l}\text { Average } \\
\text { Received } \\
\text { Base Tier } \\
(\%)\end{array}$ & $\begin{array}{l}\text { Average } \\
\text { Received } \\
\text { Enh. Tier 1 } \\
(\%)\end{array}$ & $\begin{array}{l}\text { Average } \\
\text { Received } \\
\text { Enh. Tier 3 } \\
(\%)\end{array}$ & $\begin{array}{l}\text { Average } \\
\text { Received } \\
\text { Enh. Tier 2 } \\
(\%)\end{array}$ & $\begin{array}{l}\text { Average } \\
\text { Packet } \\
\text { Delay } \\
(\mathrm{ms})\end{array}$ \\
\hline $\begin{array}{l}\text { With quality } \\
\text { Adaptation }\end{array}$ & 98 & 99 & 99 & 93 & 99 & 45 \\
\hline $\begin{array}{l}\text { Without } \\
\text { Quality } \\
\text { Adaptation }\end{array}$ & 76 & 60 & 96 & 57 & 100 & 60 \\
\hline
\end{tabular}




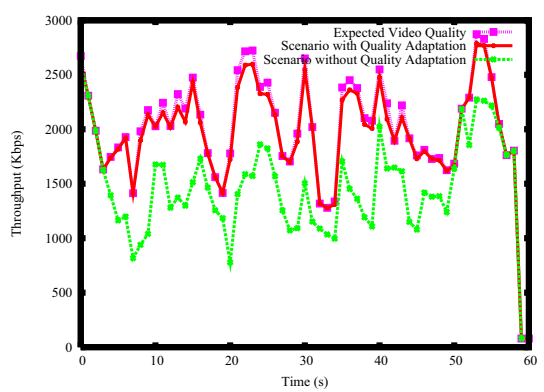

Fig. 5. Received Video Throughput

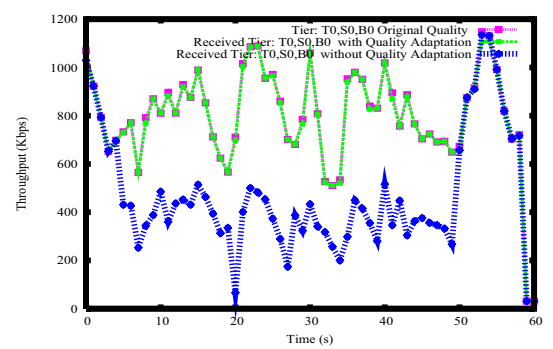

Fig. 7. (a) Base Tier

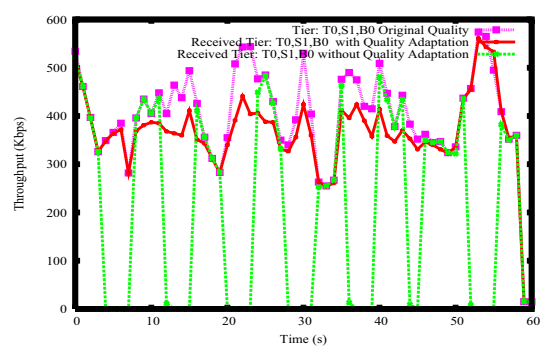

Fig. 7. (c) Enhancement Tier 2

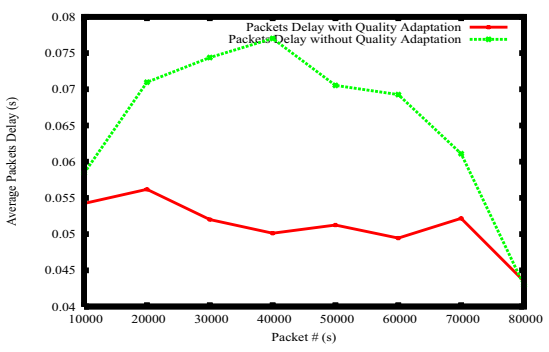

Fig. 8. 1-way Packets Delay

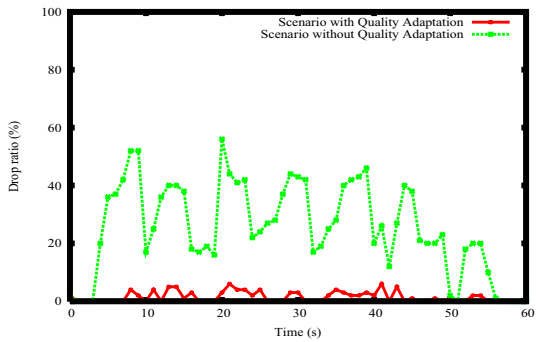

Fig. 6. Packets Drop Ratio

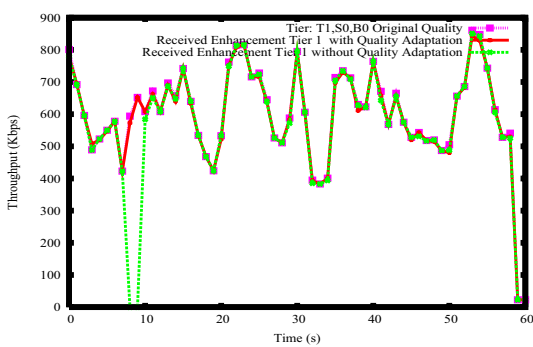

Fig. 7. (b) Enhancement Tier 1

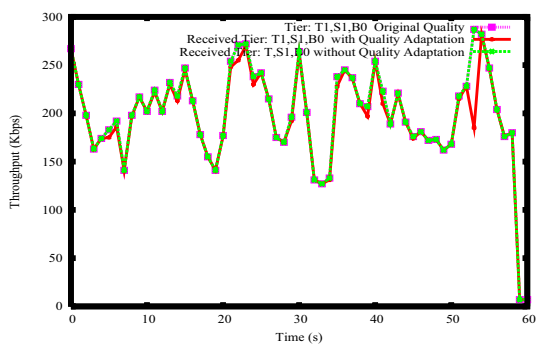

Fig. 7. (d) Enhancement Tier 3

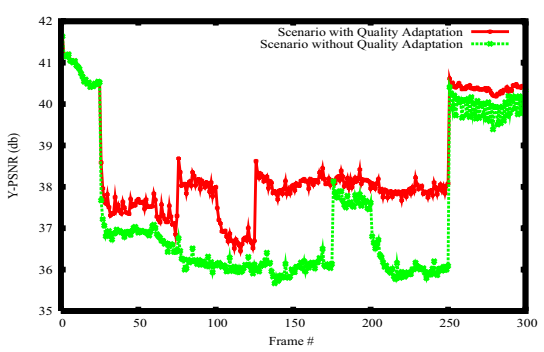

Fig. 9. PSNR 


\section{Conclusion}

In this paper, we presented a hybrid overlay network mechanism for the multimedia streaming and video on demand services in P2P environment. The hybrid overlay networks are constructed on the basis of video quality offered by sender peers and active end-to-end probing of sender peers using "RTT". The overlay organization provides a mechanism to select the best sender peers in $\Theta 1$ time. The proposed mechanism is evaluated using simulations and a significant improvement in the received throughput especially for the important video quality tiers, lower packets drop ratio for the important quality tiers, and a considerable improvement in the received packets delay are observed.

\section{References}

1. Ratnasamy, S., Francis, P., Handley, M., Karp, R., Shenker, S.: A Scalable ContentAddressable Network. In: Proc. ACM SIGCOMM, pp. 161-172. ACM Press, New York (2001)

2. Stoica, I., Morris, R., Karger, D., Kaashoek, M.F., Balakrishnan, H.: Chord: A scalable peer-to-peer lookup service for internet applications. In: Proc. ACM SIGCOMM, ACM Press, New York (2001)

3. Rowstron, A., Druschel, P.: Pastry: Scalable, distributed object location and routing for large-scale peer-to-peer systems. In: proc. of the 18th IFIP/ACM International Conference on Distributed Systems Platforms (Middleware 2001), pp. 329-350 (November 2001)

4. Zhang, X., Liu, J., Li, B., Yum, T.-S.P.: CoolStreaming/DONet: A Data-Driven Overlay Network for Efficient Live Media Streaming. In: proc. of IEEE Infocom 2005, Miami, FL, USA (March 2005)

5. Tian, R., Xiong, Y., Zhang, Q., Li, B., Zhao, B.Y., Li, X.: Hybrid Overlay Structure Based on Random Walks. In: Castro, M., van Renesse, R. (eds.) IPTPS 2005. LNCS, vol. 3640, pp. 152-162. Springer, Heidelberg (2005)

6. Crespo, A., Garcia-Molina, H.: Semantic Overlay Networks for P2P Systems, technical report (last viewed May 15, 2007), available from http://infolab.stanford.edu/ crespo/publications/op2p.pdf

7. Zhao, B., Kubiatowicz, J., Joseph, A.: Tapestry: An infrastructure for fault-tolerant widearea location and routing. Technical report UCB/CSD-01-1141, U. C. Berkeley (April 2001)

8. Vitali, A., Fumagalli, M., Cefriel: Standard-compatible Multiple-Description Coding (MDC) and Layered Coding (LC) of Audio/Video Streams, Internet Draft, Network Working Group (July 2005)

9. Schwarz, H., Marpe, D., Wiegand, T.: SNR-Scalable Extension of H.264/AVC. In: proceedings of ICIP 2004, Singapore (2004)

10. Wu, D., Hou, T., Zhang, Y.-Q.: Scalable Video Coding and Transport over Broadband Wireless Networks. In: Proceedings of the IEEE (September 2000)

11. Mushtaq, M., Ahmed, T.: Adaptive Packet Video Streaming over P2P Networks Using Active Measurements. In: ISCC 2006. proceedings of the 11th IEEE Symposium on Computers and Communications, pp. 423-428. IEEE Computer Society, Los Alamitos (2006)

12. Mahdavi, J., Floyd, S.: TCP-Friendly Unicast Rate-Based Flow Control. Technical note sent to the end2end-interest mailing list (January 8, 1997)

13. Stuart Hunter, J.: The Exponentially Weighted Moving Average. J. Quality Technology 18(4), 203-207 (1986)

14. Nash, J.: Non-Cooperative Games. The Annals of Mathematics 54(2), 286-295 (1951) 